Hybrid Metamaterials Combining Pentamode Lattices and Phononic Plates AIP/123-QED

\title{
Hybrid Metamaterials Combining Pentamode Lattices and Phononic Plates
}

\author{
A.O. Krushynska, ${ }^{1}$ P. Galich, ${ }^{2}$ F. Bosia, ${ }^{3}$ N.M Pugno, ${ }^{1,4,5}$ and S. Rudykh ${ }^{6}$ \\ 1) Laboratory of Bio-Inspired $\&$ Graphene Nanomechanics, University of Trento, \\ Trento, Italy ${ }^{\mathrm{a}}$ \\ 2) Department of Aerospace Engineering, Technion, Israel \\ 3) Department of Physics and Nanostructured Interfaces and Surfaces Centre, \\ University of Turin, Turin, Italy \\ 4) School of Engineering and Materials Science, Queen Mary University of London, \\ London, UK \\ 5) Ket Labs, Edoardo Amaldi Foundation, Italian Space Agency, Rome, \\ Italy \\ ${ }^{6)}$ Department of Mechanical Engineering, University of Wisconsin-Madison, \\ Madison, WI, USA b)
}

(Dated: 2 October 2018)

We propose a design strategy for hybrid metamaterials producing complete omnidirectional band gaps for elastic waves. The wave control in the developed metamaterials is based on simultaneous activation of scattering mechanisms in phononic plates and pentamode lattices. The approach is illustrated by numerical results for a particular hybrid configuration comprising phononic plates with cross-like cavities. We report complete band gaps of highly tunable width due to variations of geometric parameters. We show that the wave attenuation performance of the hybrid metamaterials can be further enhanced through implementation of multiphase lightweight material compositions. These give rise to omnidirectional band gaps in challenging low-frequency regions. We note that the proposed design strategy is not limited to the illustrated configurations, and can be applied to various designs of phononic plates with cavities, inclusions or slender elements.

Keywords: wave dynamics, phononic plate, pentamode lattice, wave attenuation, band gap

\footnotetext{
a) Electronic mail: akrushynska@gmail.com

b) Electronic mail: rudykh@wisc.edu
} 
Phononic and acoustic metamaterials demonstrate unusual mechanical properties ${ }^{1,2}$ and the ability to control elastic waves by producing band gaps ${ }^{3-5}$ or negative group velocity. ${ }^{6,7}$ They draw these remarkable functionalities from their engineered architectures, giving rise to remarkable dynamic characteristics at various frequency ranges. Numerous two-dimensional (2D) configurations with periodic patterns have been designed to activate wave manipulations mechanisms, resulting in omnidirectional, complete band gaps for plane-polarized elastic waves (2D band gaps). ${ }^{8}$ Applications of such designs to three-dimensional (3D) geometries are characterized by poor attenuation of oblique or normally incident waves. ${ }^{8,9}$ Common examples are phononic plates with voids ${ }^{10}$ or internal resonators ${ }^{11-13}$ with $2 \mathrm{D}$ band gaps for waves in the plane of a plate, allowing propagation of waves with out-of-plane components. ${ }^{9,13,14}$ This issue substantially limits the potential of $2 \mathrm{D}$ metamaterials for engineering applications, including seismic wave shielding, ${ }^{15,16}$ vibration mitigation, ${ }^{3,6,13}$ or wave focusing and splitting. ${ }^{17,18}$

Here, we propose the design strategy specially aimed at extension of 2D band-gap generation mechanisms in phononic plates into fully 3D settings. We show that the proposed hybrid metamaterials, based on phononic plate with pentamode lattices architectures, exhibit complete 3D band gaps due to simultaneous activation of the wave scattering in the plates and peculiar dynamics of pentamode lattices.

Pentamode lattices belong to a class of "extremal materials" introduced by Milton and Cherkaev. ${ }^{19-21}$ These essentially 3D structures consist of periodic repetitions of four tapered bars meeting at point-like joints in a diamond lattice. Ideal pentamodes have zero shear modulus, and thus exhibit fluid-like dynamics inhibiting propagation of shear waves at any frequency. ${ }^{19,22,23}$ Realistic structures are characterized by a finite, non-zero effective shear modulus. Typically, this modulus is much smaller than the effective bulk modulus. ${ }^{23,24}$ Shear and compressional waves are thus weakly coupled. This leads to frequency intervals with a single compressional mode. As we shall show, the hybrid structures formed by a combination of pentamode lattices and phononic plates can be designed to produce 3D band gaps. Such metastructures enable band gap tuning by adjusting geometrical parameters and maintain structural integrity and stability due to incorporated spheres at the joints.

A typical phononic plate has an essentially 2D configuration, if its cross-section is replicated along the thickness. This simplifies a theoretical analysis of the plate dynamics, possible optimization procedures and manufacturing process. A 2D formulation of the related 


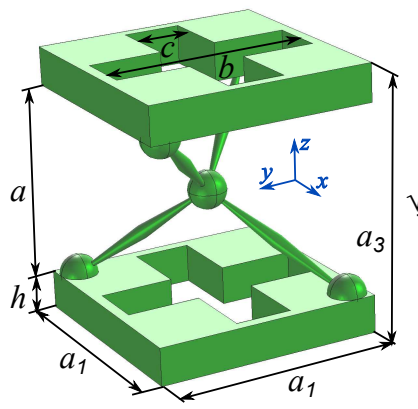

(a)
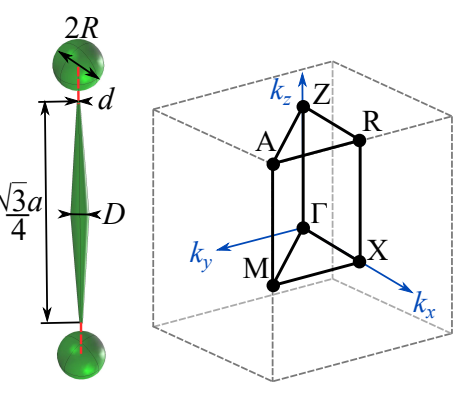

(b)

FIG. 1. (a) Metastructure unit cell; (b) irreducible Brillouin zone for a tetragonal lattice.

elastodynamic problem for the cross-sectional geometry (under assumption on an infinite thickness of the plate) enables the decoupling of motions into in-plane modes with displacements $\left\{u_{x}, u_{y}\right\}$ and out-of-plane (or transverse) modes with displacements $u_{z}$ orthogonal to the cross-sectional plane. ${ }^{8,9}$ Scattering mechanisms for these mode families are governed by a $2 \mathrm{D}$ elasticity tensor and a shear modulus, respectively, that results in 2D band gaps at different frequencies. ${ }^{9,11}$ In a 3D plate of finite thickness, the separation of modes is, in general, not possible. For waves in the cross-sectional plane, the band structures of in-plane and out-of-plane modes are superimposed; while for oblique incident waves, the two mode families become coupled, resulting in closing of 2D band gaps (see Figs.1-3 in the Supplementary Material (SM)). In order to generate complete 3D band gaps, one needs to introduce a wave attenuation mechanism in the out-of-plane direction, suppressing the coupled modes. This is typically done by designing a new 3D configuration from scratch, neglecting extensive knowledge and data available for 2D metamaterials. Our approach, in contrast, relies on preserving and using the wave attenuation abilities of $2 \mathrm{D}$ phononic plates in $3 \mathrm{D}$ hybrid metamaterials.

As an example of the proposed hybrid metamaterials, we consider phononic plates with cross-like cavities, known for their ability to induce wide 2D band gaps, ${ }^{10}$ interlayered by pentamode lattices. The metamaterial unit cell (Fig. 1a, on the left) can be periodically repeated along the $z$ axis to form a 1D meta-chain or populated along the three axial directions into a 3D structure. An extended face-centered-cubic lattice, typical for the pentamode, is replaced by a tetragonal lattice with the Brillouin zone shown in Fig. 1b. ${ }^{25}$ To maintain the structural stability, we introduce elastic spheres, connecting the lattice bars to each other and to the plates. The center and end diameters of a bar are denoted by $D$ 
Hybrid Metamaterials Combining Pentamode Lattices and Phononic Plates

and $d$, respectively; the radius of the connection spheres is $R$ (Fig. 1a, on the right). The bar length is $\sqrt{3} a / 4$, where $a$ denotes the height of the pentamode element. The cross-like cavity is defined by length $b$, width $c$, and depth $h$. The unit cell dimensions are $a_{1} \times a_{1} \times a_{3}$ with $a_{1}=a+2 R$ and $a_{3}=a+2 h$. The examples considered here are for structures made of isotropic titanium alloy Ti6A14V ${ }^{26}$ with Young's modulus $E=120$ GPa, Poisson's ratio $\nu=0.33$, and mass density $\rho=4450 \mathrm{~kg} / \mathrm{m}^{3}$.

Wave dispersion is evaluated numerically in Comsol Multiphysics 5.2 by applying BlochFloquet boundary conditions at the plate faces and solving the related eigenfrequency problem for wavenumbers along the borders of the irreducible Brillouin zone (Fig. 1b). Figure 2 shows the dispersion relation for the hybrid metamaterial with $a=16 \mathrm{~mm}, d=0.2 \mathrm{~mm}$, $D=1.2 \mathrm{~mm}, R=0.1 a, h=0.2 a, b=0.9 a_{1}, c=0.25 a_{1}$. These values are chosen to provide the widest band gaps for the transverse and in-plane modes in the pentamode lattice and the phononic plate, respectively. ${ }^{10,22}$ The color of the bands designates the mode polarization $p=\int_{V}\left|u_{z}\right|^{2} d V / \int_{V}\left(\left|u_{x}\right|^{2}+\left|u_{y}\right|^{2}+\left|u_{z}\right|^{2}\right) d V$, where $V$ is the material volume in the unit cell. Specifically, the blue color indicates in-plane modes with dominant displacements $u_{x}, u_{y}$, while the red color represents out-of-plane modes with governing displacement $u_{z}$.

The band structure diagram in Fig. 2 exhibits a complete 3D band gap highlighted in dark violet. For waves propagating along the $x-y$ plane ( $\Gamma-X-M$ directions), this band gap originates from the Bragg scattering in a phononic plate of thickness $2 h$ with the bottom and upper faces being free from stresses. Such a plate has a band gap, expanding from $55.7 \mathrm{kHz}$ to $96.4 \mathrm{kHz}$ as indicated in violet in Fig. 2. To understand the band gap formation process for waves with out-of-plane wave vector components, we note that the structure of the hybrid metamaterial resembles the lattice of a zincblende crystal with tetrahedral coordination and alternating types of masses at lattice sides. ${ }^{27}$ In this case, the wave dispersion along the $z$ axis can be approximated by a dispersion relation of a $1 \mathrm{D}$ diatomic chain. ${ }^{28}$ The chain is formed by two lumped masses (represented by a plate with half-spheres and a central sphere) connected by springs (represented by inclined bars). The solution to the corresponding dispersion relation (see SM for details) describes two dispersion bands separated by an extremely wide band gap (between $40.7 \mathrm{kHz}$ and $270 \mathrm{kHz}$, light violet region in Fig. 2). Since the real hybrid structure is formed by elastic plates with distributed, not lumped masses, the eigenmodes of these plates give rise to additional modes in the band structure (see, for example, the mode indicated by the blue circle in Fig. 2). The Bloch-Floquet conditions at 

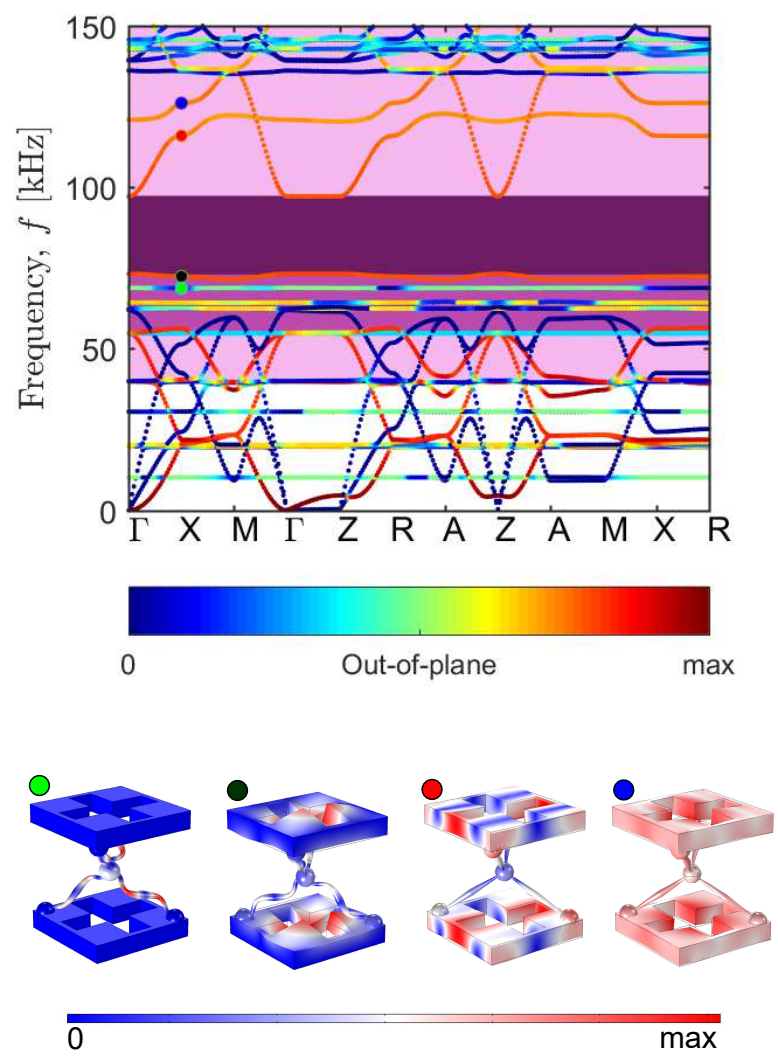

FIG. 2. Band structure diagram for the hybrid single-phase metastructure. Complete 3D band gap is shaded in dark violet; $2 \mathrm{D}$ band gap for in-plane waves in the phononic plate (without the pentamode) is highlighted in violet; 1D band gap for an equivalent diatomic chain is shown in light violet. The color of the dispersion bands indicates the mode polarization. The colored circles refer to the mode shapes at selected frequencies shown at the bottom.

the plate boundaries also generate an additional set of modes, represented, for instance, by the localized mode marked by the green circle. As a result, the 3D band gap has a narrow width, as compared to that of the diatomic chain, limited to the frequencies of the 2D band gap for in-plane modes. Mode shapes at the bounds of the 3D band gap (red and black circles) reveal strong interactions between the bars and the plates.

The introduced analogy with a diatomic chain indicates the universality of the proposed design strategy. In other words, hybrid metamaterials can be formed by any phononic plates exhibiting 2D band gaps (see e.g. Fig. 5 in the SM). This analogy also suggests that the central sphere in the pentamode unit plays an important role in the wave attenuation mechanism by the proposed hybrid designs. On the one hand, the decrease of its mass results in the shift of the upper band gap bound to higher frequencies. On the other hand, 
Hybrid Metamaterials Combining Pentamode Lattices and Phononic Plates

the smaller the radius of the sphere is, the smaller is the effective axial stiffness of the bars due to vanishing contact areas between the bars. Our simulations show that there is no band gap for $R<0.06 a$ (Fig. 6 in $\mathrm{SM}$ ); for $0.1 a \geq R \geq 0.06 a$, there exists a complete 3D band gap of almost constant width, since the variation of the mass of the central sphere remain small relative to the mass of the plate. ${ }^{29}$ Note that the revealed dependence of the band gap width on $R$ is opposite to that for pure pentamode lattices, in which wide band gaps for shear waves are obtained for vanishing contact areas between the bars. ${ }^{22}$

Figures 3a-d show the shapes of iso-frequency contours for the lowest out-of-plane mode (a-b), originating from zero frequency, and the first mode above the band gap (c-d) for the two planes of the Brillouin zone (see Fig. 1b). The symmetries of these contours reflect the rotational and reflective symmetries of the unit cell. For waves in the plane of the phononic plates, the hybrid metamaterial is isotropic, whereas for waves with non-zero components $k_{z}$, it is strongly anisotropic, regardless of frequency. The same behavior is observed for isofrequency contours of other modes as shown in Fig. 7 in the SM.

Next, we demonstrate that the 3D band gap exists in the hybrid metamaterials with a wide range of the geometric parameters, i.e. the band gap mechanism is robust and is not limited to a particular geometrical configuration. Figure 3e shows the band gap width versus height parameter $h$, while the other unit-cell parameters are fixed. The violet-shaded region indicates the frequencies of the $2 \mathrm{D}$ band gaps for the phononic plates with crosslike holes of thickness $2 h$. One can see that in most cases the $3 \mathrm{D}$ band gap occurs at the frequencies of the 2D band gaps. However, due to the interplay of different sets of modes in the hybrid metamaterials, it becomes possible to extend the 3D band gap to wider frequency ranges. Note that by varying the plate thickness, it is also possible to tune the gap width or even close the band gap, e.g., for $1.6<h<2.8 \mathrm{~mm}$. For $4 \leq h \leq 6 \mathrm{~mm}$, the bounding mode (marked with the black circle in Fig. 2) is shifted towads higher frequencies and the mode branch separates the band gap into two parts. As the thickness increases $(h>6 \mathrm{~mm})$, in-plane plate modes enter the band gap range and split it further. Similar tunability can be achieved by varying the center dimension $D$ of the bars (Fig. 8 in SM).

We further consider multi-phase configurations with different material phases for the plate and lattice units. The key idea here is to improve the structural robustness and stability by decreasing the weight of the plates, and thus, to design light-weight metamaterials. As an example, we consider a unit cell of the same structure as in Fig. 1a with the plates 
(a)

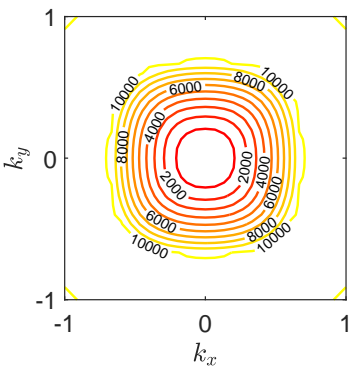

(b)
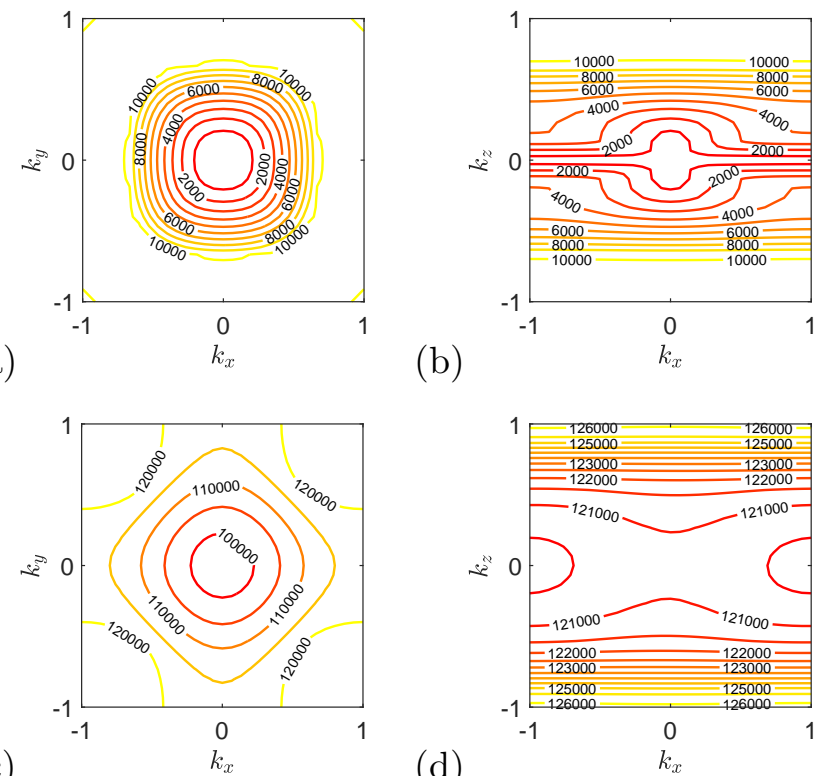

(c)

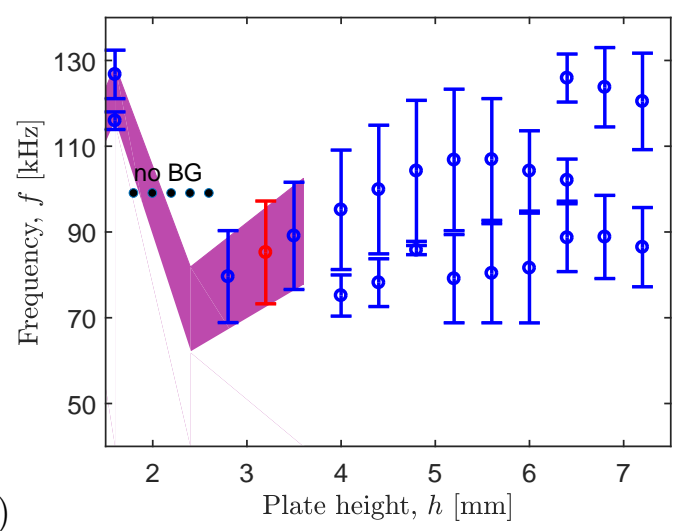

FIG. 3. (a-d) Directionality of plane wave propagation in two planes of the Brillouin zone at (a, b) low and (c, d) high frequencies. The frequencies (in $\mathrm{kHz}$ ) associated with contours are labeled. (e) The width of 3D band gaps for hybrid metamaterials with varying thickness $h$ of a phononic plate. The red bar corresponds to the band gap in Fig. 2. Shaded region shows the width of the 2D band gap for in-plane modes in the corresponding phononic plate.

made of Nylon $^{30}$ (Young's modulus $E^{(p)}=2 \mathrm{GPa}$, Poisson's ratio $\nu^{(p)}=0.41$, and mass density $\rho^{(p)}=1200 \mathrm{~kg} / \mathrm{m}^{3}$ ) and the pentamode bars made of the titanium alloy (Fig. 4a). Our simulations reveal a complete band gap of $22 \%$ gap width for $h=3.5 \mathrm{~mm}$. Note that the mid-gap frequency $22.925 \mathrm{kHz}$ is about 4 times lower than that of the corresponding single-phase configuration (Fig. 2a), and the effective material density $\rho^{\text {eff }}=270 \mathrm{~kg} / \mathrm{m}^{3}$ (evaluated as the sum of a material phase density multiplied by its volume fraction) is 3.3 
(a)

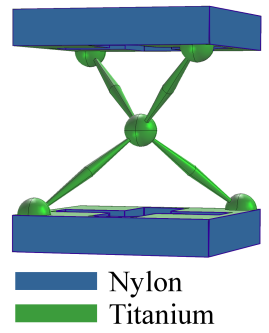

(b)
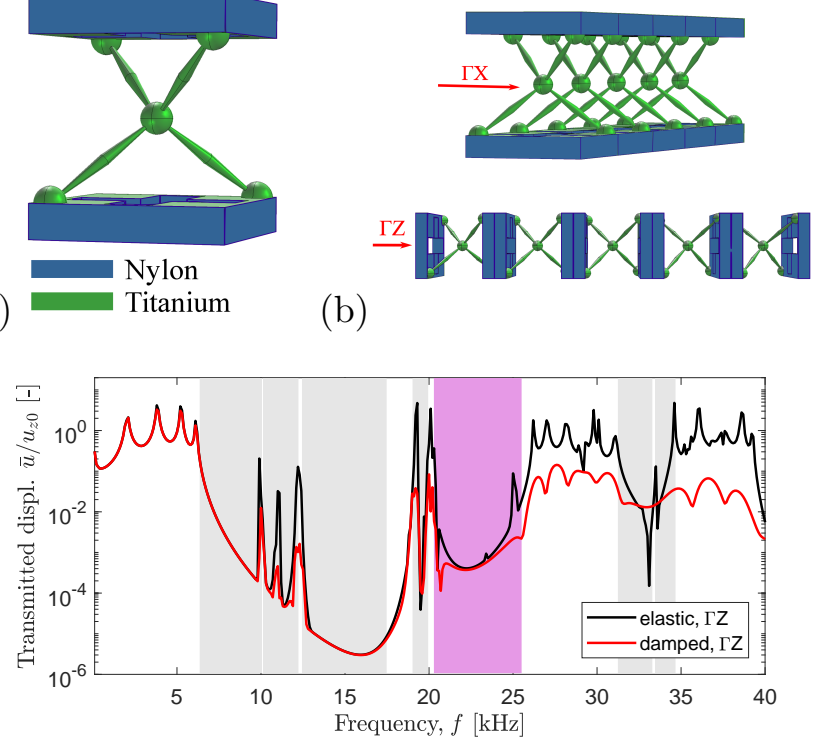

(c)

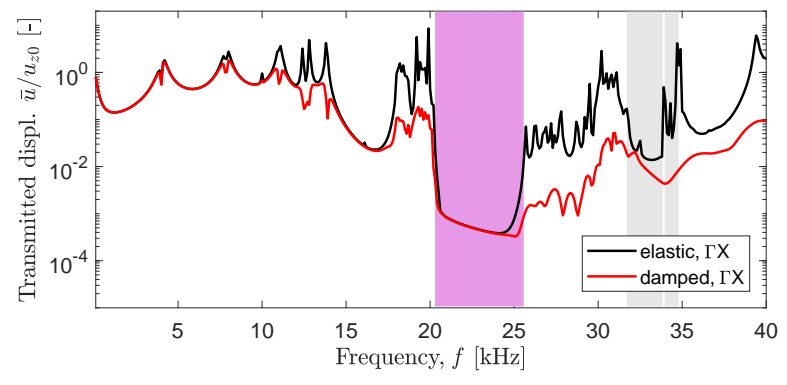

FIG. 4. (a) Unit cell of the two-phase hybrid metamaterial; (b) finite-size structures composed of five unit cells. (c-d) The normalized transmitted displacements $\sqrt{u_{x}^{2}+u_{y}^{2}+u_{z}^{2}} / u_{z 0}$ vs. frequency. Shaded regions indicate directional (gray) and complete (magenta) band gaps.

times smaller than $\rho^{e f f}=892 \mathrm{~kg} / \mathrm{m}^{3}$ for the single-phase structure, indicating a significant weight reduction. Hence, apart for the improved robustness, the two-phase light-weight hybrid configurations enable generation of the band gaps in the challenging low-frequency range. This is a distinguishing feature of these designs as compared to many other latticetype meta-structures, in which the low-frequency waves attenuation is achieved through introducing of heavy masses. ${ }^{5,26,30}$

Finally, we estimate the efficiency of wave attenuation by the two-phase configurations by performing the transmission analysis for finite-size structures. The related frequency-domain finite-element simulations are performed for 5 unit-cell samples with periodic boundary conditions at the lateral faces, which are excited by time-varying normal displacements of amplitude $u_{z 0}=1 \mu \mathrm{m}$ at one end, while the other end is attached to a perfectly matched 
layer (of 5 unit-cell size). We consider wave propagation in $\Gamma-Z$ and $\Gamma-X$ directions as shown in Fig. 4b. The curves in Figs. 4c-d represent the magnitude of normalized transmitted displacements $\sqrt{u_{x}^{2}+u_{y}^{2}+u_{z}^{2}} / u_{z 0}$ averaged upon a single unit cell. Elastic material response is denoted by black curves. The transmission drops are in a good agreement with band gap frequencies (shaded regions) or occur at frequencies of modes not excited by the applied loading (see Fig. 4b in SM). The small discrepancies can be attributed to finite sizes of the samples. Note that at the frequencies of the complete band gap (magenta shading) the transmission drops by three orders of magnitude, when the wave passes through four unit cells only. This highlights good attenuation performance of the hybrid metastructure in both of the considered propagation directions. Red lines refer to damped material behavior. The damping in Nylon is implemented using a Rayleigh model with coefficients $\alpha=1 \mathrm{~s}^{-1}$ and $\beta=4 e-7 \mathrm{~s}$, in agreement with the reported experimental data. ${ }^{30}$ For titanium, we introduce the loss factor $\eta$ in the stress-strain relation $\sigma=D(1+i \eta) \epsilon$ and assign $\eta=0.001 \mathrm{~Pa} \cdot \mathrm{s}$ corresponding to minimum experimentally measured losses. ${ }^{31}$ The magnitude of transmitted displacements in the damped case is lower compared to the elastic case. This is in agreement with the predictions for lossy composites. ${ }^{32,33}$ The amplified damping behavior at $f>25 \mathrm{kHz}$ can be explained by inapplicability of the Rayleigh model at these frequencies. ${ }^{30}$

In summary, we have proposed a design strategy for hybrid metamaterials producing 3D band gaps for elastic waves by combining phononic plates with pentamode units. We have illustrated the idea considering the example of hybrid metamaterials with a specific phononic plate and demonstrated the universality of the strategy for plates with various wave attenuation mechanisms. This paves the way to the development of numerous 3D metamaterials with target wave attenuation characteristics by exploiting, in full, the advantages of 2D configurations. For instance, one can apply powerful topology optimization techniques to design 2D geometries with required dynamic characteristics at much lower computational costs as compared to 3D cases, and then introduce them into hybrid designs with pentamode lattices by ensuring the presence of 2D band gaps.

The proposed hybrid designs involves structural robustness and stability through reinforcement of the critical joints. The performance of these metamaterials is shown to be highly tunable by varying geometric parameters, and the band gaps appear for a wide range of configurations. Thus, the wave attenuation ability relies on intrinsic property of the proposed structural design, rather than on a specific combination of geometric properties. This 
Hybrid Metamaterials Combining Pentamode Lattices and Phononic Plates

feature opens the way to the development of metamaterial configurations for broadband wave attenuation by employing rainbow-type designs with slightly varying dimensions in neighboring unit cells. ${ }^{34}$ Moreover, we have shown that multiphase designs of the hybrid metastructures can further produce low-frequency attenuation characteristics in lightweight structures. The illustrative example of polymeric plates and stiff pentamodes demonstrates the potential for a broad range of engineering applications aimed at wave and vibration attenuation. For practical realizations, further detailed studies are required, including energy localization in the slender bars, non-linear material behavior, ${ }^{35}$ and reliable experimental fabrication of the hybrid metastructures.

\section{ACKNOWLEDGMENTS}

A.K. acknowledges useful discussions with Prof. C. Yilmaz from Bogazici University in Turkey. N.M.P. is supported by the EC H2020 under the Graphene Flagship Core 2 grant No. 785219 (WP14 "Polymer Composites"), FET Proactive "Neurofibres" grant No. 732344, and "Departments of Excellence" grant L.232/2016. F.B. is supported by the EU FET Proactive "Neurofibres" grant No. 732344 and the Progetto d'Ateneo/Fondazione San Paolo "Metapp" project No. CSTO160004.

\section{REFERENCES}

${ }^{1}$ J. Christensen, M. Kadic, O. Kraft, and M. Wegener, "Vibrant times for mechanical metamaterials," MRS Commun. 5, 43462 (2015).

${ }^{2}$ S. Cummer, J. Christensen, and A. Alu, "Controlling sound with acoustic metamaterials," Nat. Rev. Mater. 1, 16001 (2016).

${ }^{3}$ S. Mohammadi, A. A. Eftekhar, A. Khelif, W. D. Hunt, and A. Adibi, "Evidence of large high frequency complete phononic band gaps in silicon phononic crystal plates," Appl. Phys. Lett. 92, 221905 (2008).

${ }^{4}$ C. Crönne, E. J. S. Lee, H. Hu, and J. H. Page, "Band gaps in phononic crystals: Generation mechanisms and interaction effects," AIP Adv. 1, 041401 (2011).

${ }^{5}$ L. D'Alessandro, E. Belloni, R. Ardito, F. Braghin, and A. Corigliano, "Mechanical low- 
Hybrid Metamaterials Combining Pentamode Lattices and Phononic Plates

frequency filter via modes separation in 3d periodic structures," Appl. Phys. Lett. 111, 231902 (2017).

${ }^{6}$ M. Lee, P. Ma, I. Lee, H. Kim, and Y. Kim, "Negative refraction experiments with guided shear-horizontal waves in thin phononic crystal plates," Appl. Phys. Lett. 98, 011909 (2011).

${ }^{7}$ V. Slesarenko, P. Galich, J. Li, N. Fang, and S. Rudykh, "Foreshadowing elastic instabilities by negative group velocity in soft composites," Appl. Phys. Lett. 113, 031901 (2018).

${ }^{8}$ Y. Pennec, J. O. Vasseur, L. D. B. Djafari-Rouhaniand, and P. A. Deymier, "Twodimensional phononic crystals: Examples and applications," Surf. Sci. Rep. 65, 229-291 (2010).

${ }^{9}$ A. Krushynska, M. Miniaci, V. Kouznetsova, and M. Geers, "Multilayered inclusions in locally resonant metamaterials: two-dimensional versus three-dimensional modeling," J. Vib. Acoust. 139, 024501 (2017).

${ }^{10}$ Y.-F. Wang and Y.-S. Wang, "Multiple wide complete band gaps of two-dimensional phononic crystal slabs with cross-like holes," J. Sound Vib. 332, 2019-2037 (2011).

${ }^{11}$ A. Krushynska, V. Kouznetsova, and M. Geers, "Towards optimal design of locally resonant acoustic metamaterials," J. Mech. Phys. Solids 71, 179-196 (2014).

${ }^{12}$ P. Wang, F. Casadei, S. Shan, J. Weaver, and K. Bertoldi, "Harnessing buckling to design tunable locally resonant acoustic metamaterials," Phys. Rev. Lett. 113, 014301 (2014).

${ }^{13}$ A. Krushynska, M. Miniaci, F. Bosia, and N. Pugno, "Coupling local resonance with bragg band gaps in single-phase mechanical metamaterials," Ext. Mech. Lett. 12, 30-36 (2017).

${ }^{14}$ Y.-F. Wang and Y.-S. Wang, "Multiple wide complete bandgaps of two-dimensional phononic crystal slabs with cross-like holes," J. Sound Vib. 332, 2019-2037 (2013).

${ }^{15}$ S. Brûlé, E. Javelaud, S. Enoch, and S. Guenneau, "Experiments on seismic metamaterials: molding surface waves," Phys. Rev. Lett. 112, 133901 (2014).

${ }^{16}$ A. Palermo, S. Krödel, K. H. Matlack, R. Zaccherini, V. K. Dertimanis, E. N. Chatzi, A. Marzani, and C. Daraio, "Hybridization of guided surface acoustic modes in unconsolidated granular media by a resonant metasurface," Phys. Rev. Appl. 9, 054026 (2018).

${ }^{17}$ R. Craster and S. Gueanneau, Acoustic Metamaterials: Negative Refraction, Imaging, Lensing and Cloaking (Berlin: Springer, 2013).

${ }^{18}$ B. Liang, J.-C. Cheng, and C.-W. Qiu, "Wavefront manipulation by acoustic metasurfaces: 
Hybrid Metamaterials Combining Pentamode Lattices and Phononic Plates

from physics and applications," Nanophotonics 7 (6), 1191-1205 (2018).

${ }^{19}$ G. W. Milton and A. Cherkaev, "Which elasticity tensors are realizable?" J. Eng. Mater. Technol. 117, 483-493 (1995).

${ }^{20}$ O. Sigmund, "A new class of extremal composites," J. Mech. Phys. Solids 48, 397-428 (2000).

${ }^{21}$ A. Norris, "Mechanics of elastic networks," Proc. R. Soc. A 470, 20140522 (2014).

${ }^{22}$ A. Martin, M. Kadic, R. Schittny, T. B uckmann, and M. Wegener, "Phonon band structures of three-dimensional pentamode metamaterials," Phys. Rev. B 86, 155116 (2012).

${ }^{23}$ T. Bückmann, N. Stenger, M. Kadic, J. Kaschke, A. Frölich, T. Kennerknecht, C. Eberl, M. Thiel, and M. Wegener, "Tailored 3d mechanical metamaterials made by dip-in directlaser-writing optical lithography," Adv. Mater. 24, 2710-2714 (2012).

${ }^{24}$ M. Kadic, T. Bückmann, N. Stenger, M. Thiel, and M. Wegener, "On the practicability of pentamode mechanical metamaterials," Appl. Phys. Lett. 100, 191901 (2012).

${ }^{25}$ R. L. Flurry, Symmetry Groups : Theory and Chemical Applications (Prentice-Hall, 1980).

${ }^{26}$ A. O. Krushynska, A. Amendola, F. Bosia, C. Daraio, N. M. Pugno, and F. Fraternali, "Accordion-like metamaterials with tunable ultra-wide low-frequency band gaps," New J. Phys. 20, 073051 (2018).

${ }^{27}$ L. Kantorovich, Quantum Theory of the Solid State: an Introduction (Dordrecht: Kluwer Academic Publishers, 2004) p. 32.

${ }^{28}$ M. I. Hussein, M. Leamy, and M. Ruzzene, "Dynamics of phononics materials and structures: historical origins, recent progress and future outlook," ASME Appl. Mech. Rev. 66, $040802(2014)$.

${ }^{29}$ The cases with $R>0.1 a$ are not considered here, as they require increasing the lateral plate dimensions that modifies the unit cell external sizes that prevents from making a fair comparison.

${ }^{30}$ L. D'Alessandro, E. Belloni, R. Ardito, A. Corigliano, and F. Braghin, "Modeling and experimental verification of an ulta-wide bandgap in 3d phononic crystals," Appl. Phys Lett. 109, 221907 (2016).

${ }^{31}$ D.-G. Lee, S. Lee, and Y. Lee, "Effect of precipitates on damping capacity and mechanical properties of ti6al4v alloy," Mater. Sci. Eng. A 486, 19-26 (2008).

${ }^{32}$ A. Krushynska, V. Kouznetsova, and M. Geers, "Visco-elastic effects on wave dispersion in three-phase acoustic metamaterials," J. Mech. Phys. Solids 96, 29-47 (2016). 
Hybrid Metamaterials Combining Pentamode Lattices and Phononic Plates

${ }^{33} \mathrm{~V}$. Slesarenko and S. Rudykh, "Harnessing viscoelasticity and instabilities for tuning wavy patterns in soft layered composites," Soft Matter 12, 3677-3682 (2016).

${ }^{34}$ S. Krödel, N. Thomé, and C. Daraio, "Modeling and experimental verification of an ulta-wide bandgap in 3d phononic crystals," Extreme Mech. Lett. 4, 111-117 (2015).

${ }^{35}$ P. Galich, N. Fang, M. Boyce, and S. Rudykh, "Elastic wave propagation in finitely deformed layered materials," J. Mech. Phys. Solids 98, 390-410 (2017). 